OPEN ACCESS

Edited by:

Nibaldo C. Inestrosa Pontifical Catholic University of Chile,

Reviewed by: Safikur Rahman,

Yeungnam University, South Korea Yolanda Diz-Chaves, University of Vigo, Spain

*Correspondence:

Zhentao Zhang zhentao104@gmail.com

Received: 27 November 2019 Accepted: 30 March 2020 Published: 14 May 2020

Citation:

Yang Y and Zhang Z (2020) Microglia and Wnt Pathways:

Prospects for Inflammation in Alzheimer's Disease.

Front. Aging Neurosci. 12:110. doi: 10.3389/fnagi.2020.00110

\section{Microglia and Wnt Pathways: Prospects for Inflammation in Alzheimer's Disease}

\author{
Yunying Yang and Zhentao Zhang* \\ Department of Neurology, Renmin Hospital of Wuhan University, Wuhan, China
}

Alzheimer's disease (AD) has been a major health issue for more than one century since it was first reported in 1906. As one of the most common neurodegenerative diseases, AD is characterized by the presence of senile plaques and neurofibrillary tangles (NFTs) in the affected brain area. Microglia are the major regulators of neuroinflammation in the brain, and neuroinflammation has become recognized as the core pathophysiological process of various neurodegenerative diseases. In the central nervous system (CNS), microglia play a dual role in $A D$ development. For one thing, they degrade amyloid $\beta(A \beta)$ to resist its deposition; for another, microglia release pro-inflammatory and inflammatory factors, contributing to neuroinflammation as well as the spreading of $A \beta$ and tau pathology. Wnt pathways are important regulators of cell fate and cell activities. The dysregulation of Wnt pathways is responsible for both abnormal tau phosphorylation and synaptic loss in AD. Recent studies have also confirmed the regulatory effect of Wnt signaling on microglial inflammation. Thus, the study of microglia, Wnt pathways, and their possible interactions may open up a new direction for understanding the mechanisms of neuroinflammation in $A D$. In this review, we summarize the functions of microglia and Wnt pathways and their roles in $A D$ in order to provide new ideas for understanding the pathogenesis of $A D$.

Keywords: Alzheimer's disease, microglia, neuroinflammation, Wnt pathway, AD pathology

\section{INTRODUCTION}

\section{Microglial Activation and Immune-Inflammatory Response in AD Development}

Amyloid-beta $(\mathrm{A} \beta)$ deposition, abnormal tau phosphorylation, and synaptic dysfunction are the characteristic pathological changes of AD (Figure 1; Robakis et al., 1987; Baas and Black, 1990; Wang et al., 1996; Klyubin et al., 2005; Shankar et al., 2008; Kuhn et al., 2010; Martin et al., 2013a,b; Pester et al., 2013; Zhang et al., 2014, 2018; Willem et al., 2015; Roostaei et al., 2017; Kreutzer and Nowick, 2018; Trillaud-Doppia and Boehm, 2018; Baas and Qiang, 2019). Tightly connected with $\mathrm{AD}$ pathology, microglia and microglial inflammation work actively in $\mathrm{AD}$ onset and progression. Secretion and phagocytosis are the most studied functions in microglial neuroinflammation. This is not only because microglia are the dominant immune cells releasing inflammatory or pro-inflammatory factors in the central nervous system; they also interact with other brain cells by secreting cytokines (Yang et al., 2011; Fricker et al., 2018). More importantly, 


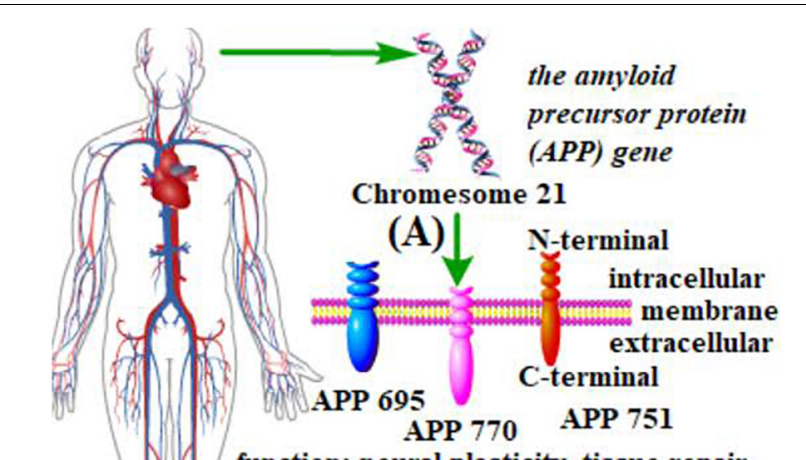

function: neural plasticity, tissue repair

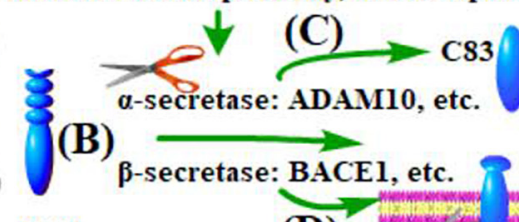

APP

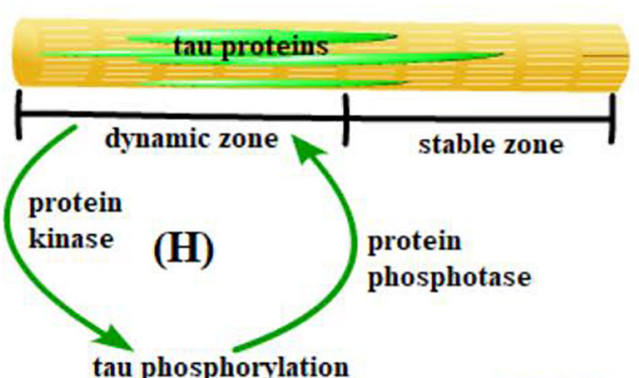

microtubule

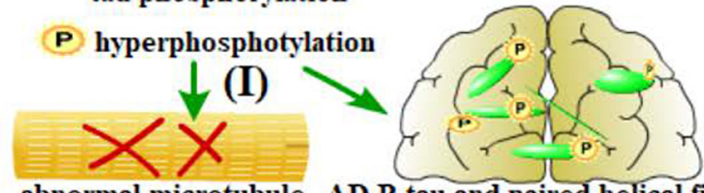

abnormal microtubule AD P-tau and paired helical filament tau

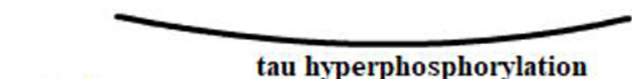

tau hyperphosphorylation

\section{fibrils}

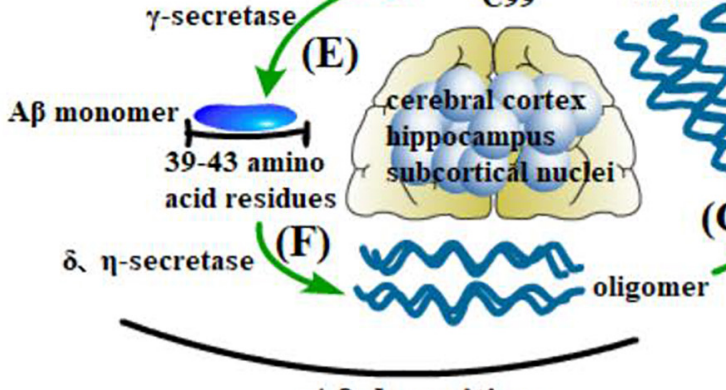

A $\beta$ deposition

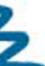

early stage hallmark

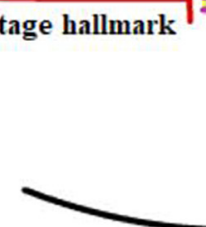

synapse dysfunction and cognitive impairment

FIGURE 1 | Pathological changes in AD. (A-G) A $\beta$ deposition and synapse dysfunction: the amyloid precursor protein (APP) gene generates at least three isomers of APP proteins in the brain. $\beta$-secretase cleaves APP into the C-terminal fragment C99, which is sequentially processed by $\gamma$-secretase to produce A 3 . In contrast, $\alpha$-secretase cleaves APP into C83, which inhibits A $\beta$ deposition. Under pathological conditions, the soluble A $\beta$ monomer turns into oligomers and insoluble fibers, with the former being more pathogenic. Existing in the cerebral cortex, hippocampus, and some subcortical nuclei, these anomalously aggregated ABs cause synapse dysfunction, thus leading to cognitive impairment. (H,I) Tau protein phosphorylation: microtubules have both dynamic and stable zones, and tau mostly exists in the former. As major microtubule-associated proteins in nerve cells, the phosphorylation and dephosphorylation of tau proteins are controlled by protein kinases and protein phosphatases (PP), respectively. Abnormality of these processes will cause an aberrant structure of microtubules. In $A D$ brains, two aberrant forms of tau proteins (AD P-tau and paired helical filament tau) are the main components of NFTs.

various immunological receptors expressed on microglia have equipped them with the capability to proliferate, accumulate, change morphology, and, especially, phagocytose $A \beta$, so they function as both neuron protectors and injurers with activated neuroinflammation in $\mathrm{AD}$ development (Füger et al., 2017; Ayata et al., 2018). For example, CD22, an immunological receptor on microglia, has been newly proved to affect microglial phagocytosis to the greatest extent in aging brains (Pluvinage et al., 2019). As well as phagocytosis-related receptors, TREM2 is another important receptor on microglia that maintains cell function in $\mathrm{AD}$ and has been extensively studied lately from both the gene and protein level (Krasemann et al., 2017;

Abbreviations: AD, Alzheimer's disease; NFTs, neurofibrillary tangles; CNS, central nervous system; $A \beta$, amyloid $\beta$; APP, amyloid precursor protein; PP, protein phosphatases; Dkk1, Dickkopf-1; Gsk3 $\beta$, glycogen synthase kinase 3; FZD, Frizzled; LRP5/6, low-density lipoprotein receptor-related protein 5/6; Dvl, Disheveled; PCP, planar cell polarity; NPCs, neural progenitor cells; SVZ, subventricular zone.
Shi et al., 2017; Filipello et al., 2018; Sayed et al., 2018; Long et al., 2019; Yin et al., 2019; Zhou et al., 2019). TREM2 can inhibit $\beta$-catenin degradation while stabilizomg $\beta$-catenin, thus activating the canonical Wnt pathway to act protectively in AD. TREM2 mutations will lead to abnormality of Wnt/ $\beta$-catenin signaling and microglial dysfunction, which causes high risk of $\mathrm{AD}$ (Zheng et al., 2017; Meilandt et al., 2020). Thus, Wnt pathways and microglial functions may be the targets of some newly found genes that contribute to $\mathrm{AD}$. The $\mathrm{Wnt} / \beta$-catenin pathway will be described in detail in section Wnt Pathway Regulation Is Promising in $\mathrm{AD}$ Development.

\section{Wnt Pathway Regulation Is Promising in AD Development}

Wnt signaling forms a vast network in the development, regeneration, and protumor pathogenesis of all the vital organs 
in human body (Watson et al., 2013; Kim et al., 2017; Patel et al., 2017; Shen et al., 2017; Ma et al., 2019). In the CNS, Wnt signals contribute to vascular repair and the development of the blood-brain barrier and share communications with microglial neuroinflammation (Hübner et al., 2018; Van Steenwinckel et al., 2019). Wnt signals are composed of one canonical pathway (Wnt/ $\beta$-catenin) and two non-canonical pathways (Wnt/PCP and $\mathrm{Wnt} / \mathrm{Ca}^{2+}$ ), as well as their cascade molecules. Targeting AD pathology, all these pathways are indicated in $\mathrm{AD}$ development (Elliott et al., 2018; Amal et al., 2019; Petrache et al., 2019).

The canonical Wnt pathway centers on the core molecule $\beta$-catenin. $\beta$-catenin can be normally degraded through ubiquitination, while Wnt3a, a member of the canonical pathway, can stabilize $\beta$-catenin. When Wnt proteins of canonical families combine with their receptors FZD and LRP $5 / 6$ on microglia, $\beta$-catenin then accumulates in the plasma, transfers to the nucleus, and binds to the TCF/LEF transcription factor, thus initiating the transcription of the targeted genes (Halleskog et al., 2011; Gray et al., 2013; Ren et al., 2015). This activation of the Wnt canonical pathway changes the immune-phenotype of microglia and brings microglia into an activated and pro-inflammatory state, which is symbolized by high expression of IBA1, CD68, CD33, and CD11b (i.e., CR3) (Halleskog and Schulte, 2013; Mrdjen et al., 2018; Böttcher et al., 2019; O'Koren et al., 2019). Microglial activation then alleviates $\mathrm{AD}$ pathology, and this again proves the dual role microglial neuroinflammation plays in $\mathrm{AD}$ development, since neuroinflammation is not completely negative for protecting normal brains from AD (Toledo and Inestrosa, 2010). However, according to the literature available at present, whether Wnt signaling has a direct influence on microglial phagocytosis remains unknown; microglial pro-inflammatory transmission is currently thought to be the main target that Wnt signaling relies on to exert effect (Song et al., 2019; Van Steenwinckel et al., 2019). There is no doubt, however, that microglial phagocytosis is always followed by the presence of inflammation. Therefore, investigating not only the Wnt-related microglial inflammation but also the effect of Wnt signaling on the other microglial functions such as phagocytosis is of great significance. Although there is no evidence supporting the direct interaction between $\mathrm{A} \beta$ and Wnt proteins, $\mathrm{A} \beta$ is believed to promote $\beta$-catenin phosphorylation and cause $\beta$-catenin degradation and to still be able to block Wnt signaling in an indirect way by combining with the cysteine-rich region of Wnt receptor FZD (Chen and Bodles, 2007; Magdesian et al., 2008; He and Shen, 2009). In AD models, a sharp decrease of $\beta$-catenin and $A \beta$ overproduction inhibit the canonical Wnt pathway and further decrease Wnt receptors (Liu et al., 2014; Elliott et al., 2018).

The two non-canonical Wnt pathways (members: Wnt11, Wnt5a, Wnt7b, Wnt9b, etc.) are independent of $\beta$-catenin (Figure 2) and are best known for regulating cell fate (differentiation, proliferation, etc.) and cell activities (polarization, migration, etc.) (Rosso et al., 2005; Ciruna et al., 2006; Carmona-Fontaine et al., 2008; Farías et al., 2009; Ishida-Takagishi et al., 2012; Florian et al., 2013; Scholz et al., 2016; Chavali et al., 2018). Wnt5a, one of the members of the non-canonical Wnt pathways, is also proved to contribute to microglial inflammation and AD development (Farías et al., 2009; Kitazawa et al., 2011; Killick et al., 2014; Sellers et al., 2018), the underlying mechanism of which has not been discovered.

In summary, the activation of canonical and non-canonical pathways can both lead to microglial inflammation, with the former playing a protective role in $\mathrm{AD}$ development and the latter contributing to $\mathrm{AD}$ development. More often than not, whilst the canonical pathway is restrained, the non-canonical one will be activated. The inhibitor of the canonical pathway and activator of non-canonical pathways, Dickkopf-1 (Dkk1), which is produced by $A \beta$ induction, is proved to cause synaptic toxicity (Killick et al., 2014). In contrast, Fasudil, the inhibitor of the non-canonical Wnt pathways, is proved to effectively reverse the unbalance between canonical and non-canonical Wnt pathways and reduce the synaptic toxicity caused by $\mathrm{A} \beta$ (Sellers et al., 2018). However, the mechanism by which the shift from canonical to non-canonical Wnt pathway transmits to alter the role of microglia from immune executors to neuron injurers is still under investigation (Figure 3). It can be speculated that Wnt signaling, no matter whether from inside microglia or from other brain cells, must affect the cell fate, activities, or functions of microglia to influence microglial neuroinflammation and thus be involved in $\mathrm{AD}$, possibly at the transcriptional, gene, or molecule level. Controversially, some research has adopted interventions to attenuate microglial inflammation via activating the canonical Wnt pathway, which has added complexity to the determination of the relationship between the canonical Wnt pathway and microglial inflammation (Chen et al., 2017; Jiang et al., 2017; Bhat et al., 2018; Yao et al., 2019; Zhang D. et al., 2019).

\section{MICROGLIA ARE MORE THAN IMMUNE CELLS IN THE CENTRAL NERVOUS SYSTEM}

The existing literature has confirmed the regulatory effect of Wnt pathways on microglial inflammation, and Wnttargeted interventions such as Fasudil are promising in AD treatment. However, these results are far from explaining in detail the intermediate elements prior to the ultimate proinflammatory effect, which gives rise to limitations to the development of Wnt-targeted or microglia-pertinent therapies for treating $\mathrm{AD}$. These intermediate elements cover three aspects: cell fate (microglial development in the CNS), cell activities (polarization, migration, and correlations with other cells) and cell functions (phagocytosis, release of inflammatory factors, refinement of synapses, and microglial genes affecting functions) of microglia. Therefore, attention has been focused on explaining how Wnt signaling affects the three aspects or follows other mechanisms, thus regulating microglial neuroinflammation in $\mathrm{AD}$ development. In sections Microglia Are More Than Immune Cells in the Central Nervous System, Microglial A $\beta$ Phagocytosis-Degradation-Dissemination and Concomitant Inflammation, and Wnt promotes Microglial Inflammation in AD Development, we will discuss the important aspects around microglia in the hope of finding the potential targets of Wnt signaling on microglia. 


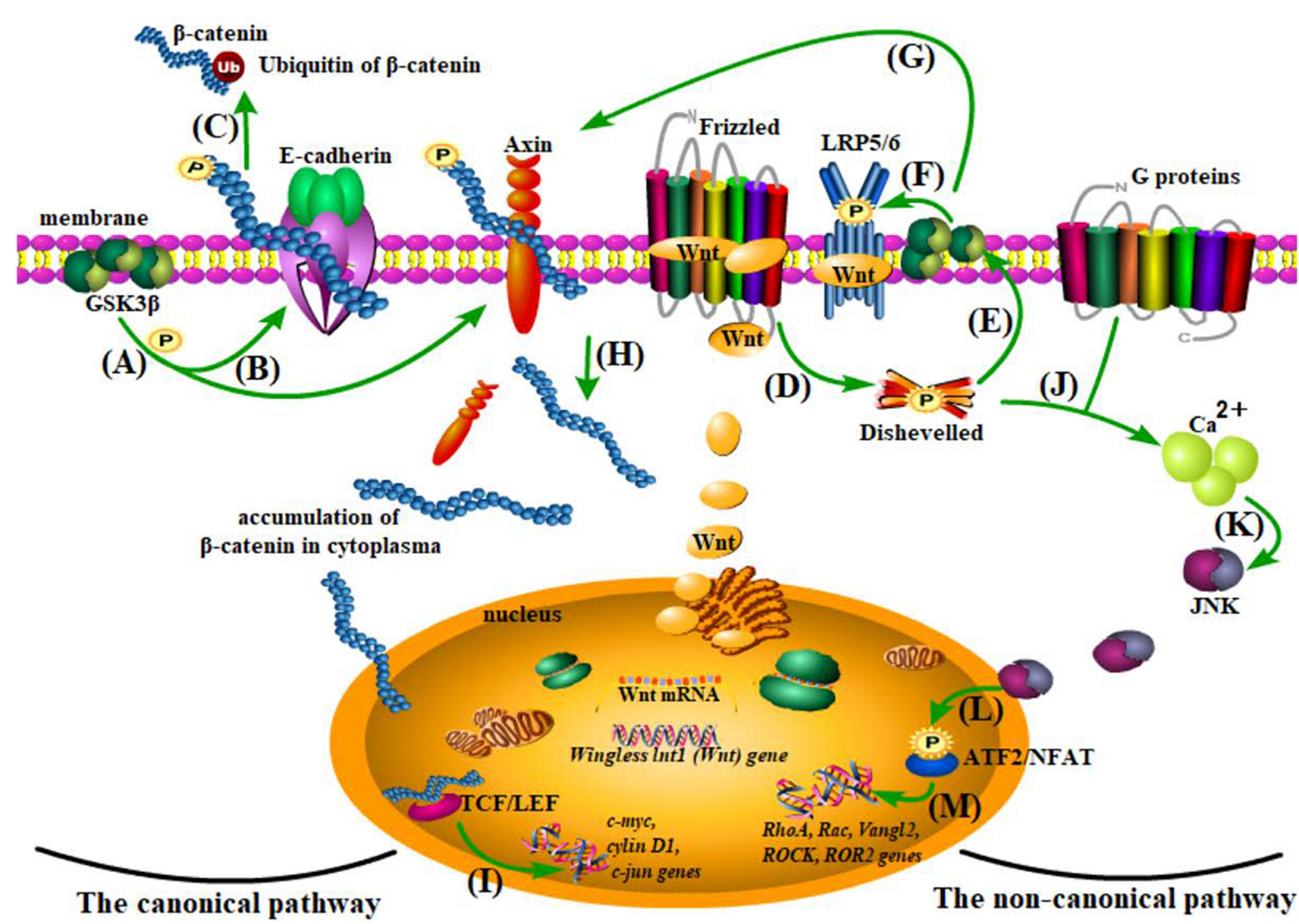

FIGURE 2 | The Wnt pathways. (A-C) $\beta$-catenin degradation: under normal conditions, $\beta$-catenin is anchored to the cell membrane with E-cadherin and Axin and goes to ubiquitination after being phosphorylated by glycogen synthase kinase 3 (Gsk3ß). (D-I) The canonical pathway: when Wnt combines with the cysteine-rich region of its receptor protein, Frizzled (FZD), and the coreceptor-low density lipoprotein receptor-related protein 5/6 (LRP5/6) acts on the Disheveled (Dvl) protein and inhibits Gsk3 $\beta$, thus separating $\beta$-catenin from Axin, the accumulated $\beta$-catenin from plasma to nucleus is capable of enhancing the activity of TCF/LEF (a transcriptional factor) and therefore influencing gene transcription of c-myc, cylin D1, c-jun, etc. (J-M) The non-canonical pathway: the activation of the Wnt/planar cell polarity (PCP) and the Wnt/Ca ${ }^{2+}$ pathways, although independent of $\beta$-catenin, share the initial process (Wnt combines with receptors and activates Dvl) with the canonical pathway. With the assistance of $\mathrm{G}$ proteins, $\mathrm{Ca}^{2+}$ is recruited in the plasma to cause cascade reactions and finally affect transcription of $R a c, R h o A$, Vangl2, ROCK, and ROR2.

\section{Microglia Participate in CNS Development, Conduct Synapse Modification, and Crosstalk With Wnt Signaling}

The development of the CNS can hardly occur without microglia. During brain development, microglia will release growth-promoting substances to assist the neurogenesis of the hippocampus and cortex and promote the maturity of neural progenitor cells (NPCs) via certain physiological processes (Choi et al., 2008; Sierra et al., 2010; Ueno et al., 2013). Conversely, NPCs can guide the migration and accumulation of microglia in the subventricular zone (SVZ) (Arnò et al., 2014). However, the relationship between microglia and neurons is much more complex than that: the effect microglia have on synapses and neural circuits is multilevel. From the perspective of neurogenesis, microglia can act as synapse pruners by way of phagocytosis based on some immunological receptor pathways, the representative one of which is the CR3/CX3CL1 molecule pair on microglia (Barger and Basile, 2001; Paolicelli et al., 2017;
Gunner et al., 2019). Under the intervention of harmful stimulus, the chemokine CX3CL1 is transformed by ADAM10 into its secretory form, interacts with its receptor Fractalkine specifically expressed on the neurons, and thus protects synapse function. This physiological refinement is conducive to maintaining the normal number of synapses and promoting the maturation of neuronal circuits (Schafer et al., 2012). When the canonical Wnt pathway is inhibited, Fractalkine expression will undergo a sharp disease, thus causing synapse degeneration (Gunner et al., 2019; Ru et al., 2019). This corresponds with the fact that Wnt3a (a member of the canonical Wnt pathway) upregulation and Wnt5a (a member of the non-canonical Wnt pathways) downregulation promote neurogenesis (Joseph et al., 2017; Bhat et al., 2018). Therefore, Wnt signaling may play a role in microglial synapse modification, and whether this sequentially contributes to synchronous microglial inflammation is worth investigating.

From the other perspective, when the CNS is confronted with neurodegenerative events such as during $\mathrm{AD}$ development, the abnormal expression of tau protein can enhance the 


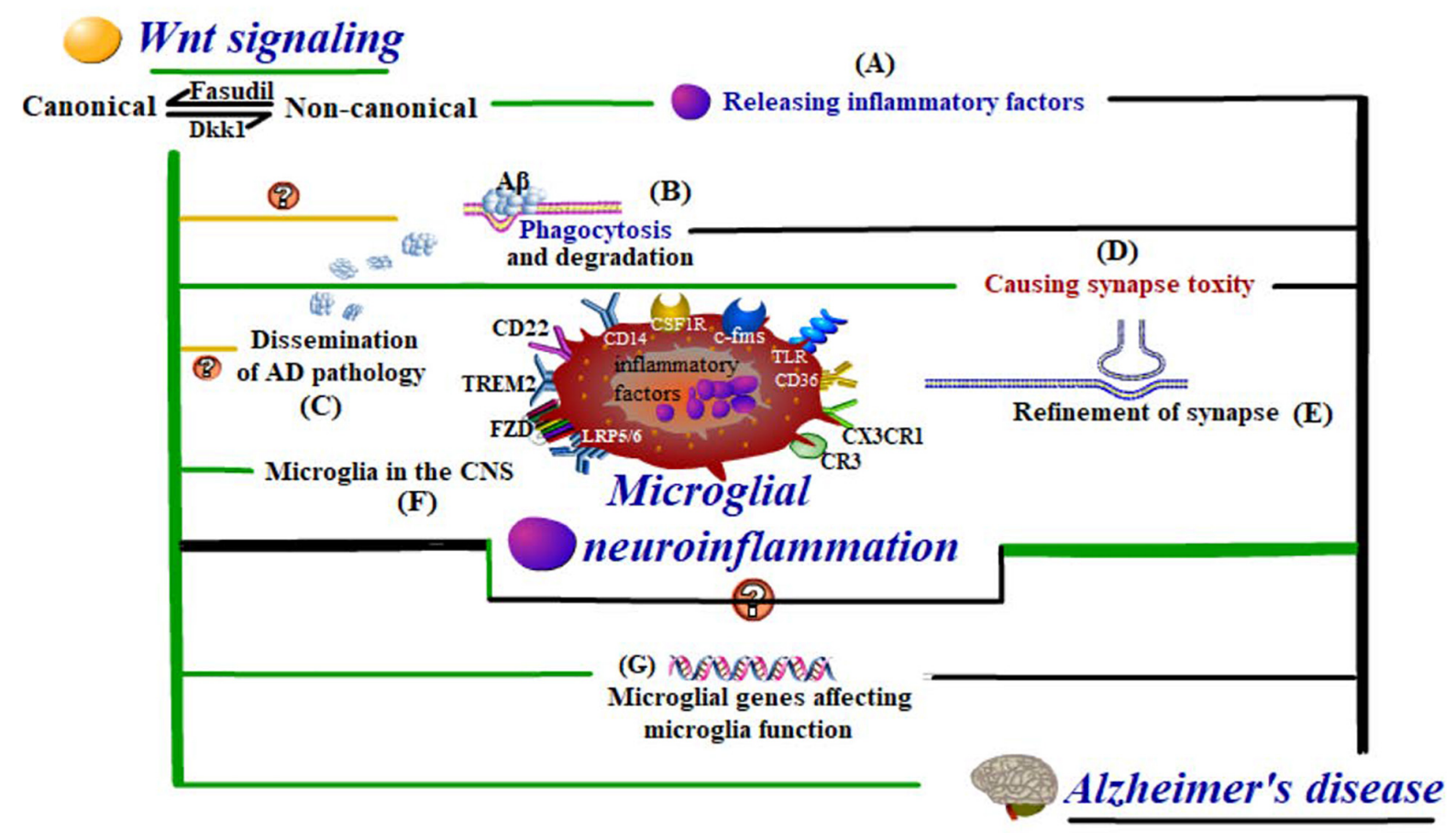

FIGURE 3 | Wnt-regulated microglial neuroinflammation in Alzheimer's disease. Wht regulation influences the cell fate and activities of (F) microglia in the CNS, even at the level of (G) microglial genes affecting microglial functions. These functions include (A) inflammatory response, (B) A $\beta$ phagocytosis and degradation, (C) dissemination of $A D$ pathology, and (D,E) synapse modification, which are all closely related to neuroinflammation in $A D$ development.

combination of the complement fragment $\mathrm{Clq}$ and synapses and, as a result, over-activate microglial phagocytosis of synapses (Dejanovic et al., 2018; Bie et al., 2019). A $\beta$ is sure to cause synaptic toxicity and then be phagocytosed by microglia when $\mathrm{A} \beta$ itself accumulates to a higher concentration, the mechanism of which will be discussed concretely in section Microglial A $\beta$ Phagocytosis-Degradation-Dissemination and Concomitant Inflammation. Once the synapses are damaged by $\mathrm{A} \beta$, they will be eliminated by microglia. Meanwhile, during this process, deleterious substances produced from microglia can simultaneously induce synapse dysfunction, which is the pathological foundation of cognitive impairment in $\mathrm{AD}$ and, to some extent, decides the $\mathrm{AD}$ plaque burden a patient can bear (Robinson et al., 2014; Turano et al., 2015; Edwards, 2019). These deleterious substances produced from microglia are from at least two sources: one is the harmful compounds released during microglial synapse elimination, while the other is the inflammatory factors delivered in step with the process of microglial A $\beta$ phagocytosis (Jiang et al., 2016). As a matter of fact, $A \beta$ not only damages synapses directly in this way but it also combines with the Wnt receptor FZD, thus causing the dysfunction of the canonical pathway (Magdesian et al., 2008). What makes this inhibition effect worse is that $A \beta$ can also induce the production of the powerful inhibitor of the canonical Wnt pathway, Dkk1, which further inactivates this protective pathway against AD (Killick et al., 2014). This no doubt will aggravate the microglial inflammation and synapse damage, which is detrimental for reversal of $\mathrm{AD}$, because the microglial synapse modification is the initiator rather than the consequence of $\mathrm{AD}$ and happens as early as in the initial stage of $\mathrm{AD}$, just before NFT formation (Yoshiyama et al., 2007; Lui et al., 2016; Sellgren et al., 2019).

\section{Regeneration vs. Degeneration: Wnt-Regulated Microglial Activities in AD}

So far, we have shown that despite microglia not being constitutive components of the CNS, they are still closely tied with NPCs, neurons, and other glial cells (Xiong et al., 2016). As immunological executors in the CNS, microglia are more than immune cells (Morizane et al., 2017). Since the merit of Wnt pathways in tissue regeneration has been widely noticed, microglia also play a role in neural regeneration through their correlations with neurons, oligodendrocytes, and astrocytes (Dou et al., 2012; Li et al., 2012; Slotkin et al., 2017; Wlodarczyk et al., 2017; Giera et al., 2018; Hill et al., 2018; Bellver-Landete et al., 2019). Even in an ALS model, astrocytes are considered to promote microglial proliferation along with neural protective effects in a Wnt-dependent way (Ouali Alami et al., 2018). Although the canonical Wnt pathway and proper content of microglial activation act protectively in neurogenesis, these two protective effects are not always in step with each other (Bhat et al., 2018). Some extreme situations can better prove these correlations. The Wnt3astimulated glioblastoma cells, which can be seen as the result of overaction of the canonical Wnt pathway, show greater 
invasiveness than general glioblastoma and greater microglial infiltration when co-cultured with microglia (Matias et al., 2019). In this case, Wnt3a is again proved to be able to change the immune phenotype of microglia, which is likely to produce inflammation rather than promote neural repair (Matias et al., 2019; Song et al., 2019; Van Steenwinckel et al., 2019). These results have demonstrated that Wnt signaling is a double-edged sword to microglial fate and activities, which is similar to the fact that microglial neuroinflammation is also a double-edged sword in neurodegenerative diseases, including $\mathrm{AD}$. In the cases of cell aging and neurodegenerative diseases, microglia will change into an activated state, resisting pathological alternation of these diseases while simultaneously accelerating neurodegeneration via immune and inflammatory response (Davies et al., 2017; Gibson et al., 2019). These immune responses show different levels of severity in different brain regions, with the cerebellum and hippocampus being most sensitive, which may account for the phenomenon that some pathological proteins specifically concentrate on certain brain regions (Grabert et al., 2016). This bidirectional effect of Wnt pathways on microglia and the multilevel effect of microglial inflammation on $\mathrm{AD}$ development require us to adopt appropriate interventions to regulate Wnt pathways, in expectation of controlling the microglial activation and inflammation at the level of neural repair and protection rather than neurodegeneration.

\section{Exploring Elements Affecting Microglial Functions at the Gene Level and Gene-Level Wnt Regulation}

At the end of section Regeneration vs. Degeneration: Wnt Regulated Microglial Activities in AD, we put forward a goal of Wnt regulation, which involves looking for a balance of Wntregulated microglial inflammation in $\mathrm{AD}$. The complexity of this goal lies in that microglia share an extensive connection with neurons and other glial cells in the CNS, which means that microglial inflammation participates not only in $\mathrm{AD}$ but in other neurodegenerative diseases. Moreover, Wnt signaling itself has a wide range of effects inside the human body. However, the present studies concerning the relation between Wnt signaling and microglia have not covered the non-canonical Wnt pathways, and even the regulation of the canonical pathway on microglia is still stagnating at the level of biological effect (proinflammatory effect). To make this goal feasible, strict regulation at the molecular level, gene level, and even transcriptional level is greatly needed. No matter what the microglial biological effects are, modifying synapses or promoting inflammation, these effects are closely related to phagocytosis and other microglial functions, which are based on the corresponding immunological receptors or other molecules. These biological factors also affect the microglial function at the gene level, the effect of which will be greatly disturbed when certain gene mutations happen in microglia (Schafer et al., 2016; Nagarajan et al., 2018). TREM2 is such an important receptor on microglia, maintaining cell function and affecting $\mathrm{A} \beta$ processing in $\mathrm{AD}$ (Ulland et al., 2017). This receptor is capable of stabilizing $\beta$-catenin, thus activating the canonical Wnt pathway to act protectively in AD. TREM2 mutation or deletion will lead to abnormality of Wnt/ $\beta$-catenin signaling and microglial dysfunction, which causes a high risk of AD (Zheng et al., 2017; Meilandt et al., 2020). What is more, the mutation of the classical AD-susceptible gene PSEN was proved early on to be followed with $\beta$-catenin upregulation (Zhang et al., 1998). Aside from this, with the increase in focus on the biological effects of competing endogenous RNA (ceRNA, a collection of non-coding RNAs over 200nt interacting with mRNA, thus influencing gene expression, with miRNA, rRNA, lncRNA, circRNA, etc. included), recent studies have explored the Wnt regulation on microglia affected by some lncRNA, which opens up a new direction for studying Wnt regulation on microglia at the gene level (Xia et al., 2017; Ross et al., 2018; Cherubini et al., 2019; Han and Zhou, 2019; Zhang L. et al., 2019). Considering that many neurodegenerative diseases possess genetic susceptibility where genes concerning microglial functions are involved, the pathological changes of various neurodegenerative diseases related to these genes and corresponding microglial functions are presented in Table 1. These may provide possible targets for Wnt regulation on microglia at the gene level (Chrétien et al., 2004; Wiendl et al., 2005; Baker et al., 2006; Bensinger and Tontonoz, 2009; Llorens et al., 2014; Karch and Goate, 2015; Markovinovic et al., 2017; Aliseychik et al., 2018; Conway et al., 2018; Rui et al., 2018; Crotti et al., 2019; Estus et al., 2019; Filippini et al., 2019; Henstridge et al., 2019; Huang et al., 2019; Sakae et al., 2019).

\section{MICROGLIAL A $\beta$ PHAGOCYTOSIS- DEGRADATION-DISSEMINATION AND CONCOMITANT INFLAMMATION}

Except for regulating synaptic functions, microglia are also capable of regulating $A \beta$ deposition by phagocytosis. Since microglia share an extensive connection with neurons based on some special extracellular micro-vesicles, the proper processing of $A \beta$ by microglial phagocytosis seems to be of great significance in that microglial dysfunction will also be harmful for neurons (Budnik et al., 2016; Vogel et al., 2018; Wheeler et al., 2018; Klein et al., 2019). A high concentration of $A \beta$ has a direct toxic influence on neurons, while a low concentration induces the exposure of signals on neurons and attracts microglia to phagocytose and degrade $A \beta$ (Neniskyte et al., 2011; Wendeln et al., 2018). Under $A \beta$ induction, neurons release endogenous substances to combine with their upregulated receptors on microglia, which is followed by pro-inflammatory events such as inflammasome accumulation in microglia (Du Yan et al., 1997; Olmos-Alonso et al., 2016). This is followed by the release of inflammatory factors like IL-1 $\beta$, IL-33, and COX2, which recruits much more microglia with increasing production of other neurotoxic factors (Halle et al., 2008; Johansson et al., 2015; Fu et al., 2016; Lee J.Y. et al., 2018). Under chemotaxis, these microglia migrate to and surround their targets $-\mathrm{A} \beta$ and tau and engulf them through special pathways and receptors such as CD14 and CD36 (Liu et al., 2005; Lucin et al., 2013; Chen et al., 2016; Jiang et al., 2016; Martin et al., 2017; Stancu et al., 2019). The inflammatory factors produced during this period 
TABLE 1 | Microglial genes contributing to neurodegenerative diseases.

\begin{tabular}{|c|c|c|c|}
\hline Diseases & Microglial genes & Mechanism pathology/function & Main clinical symptoms \\
\hline \multirow[t]{10}{*}{ Alzheimer's disease (AD) } & TREM1/2 & $\begin{array}{l}\text { Immune response, } A \beta \text { phagocytosis, synapse } \\
\text { modification and related to tau pathology }\end{array}$ & Cognitive impairment \\
\hline & APOE4 & Lipid metabolism, $A \beta$ deposition & \\
\hline & PLCG/ABI3 & Immune response, $A \beta$ pathology & \\
\hline & PSEN & Immune response, Wnt pathway & \\
\hline & PTK2B/PICALM/CR1 & Endocytosis, $A \beta$ metabolism & \\
\hline & CD22/14/36/33 & Immune response, $A \beta$ phagocytosis & \\
\hline & HLA & Immune response & \\
\hline & BIN1 & Endocytosis, tau pathology & \\
\hline & CR3 & Immune response, synapse elimination & \\
\hline & $\mathrm{HDAC} 1 / 2$ & $A \beta$ phagocytosis & \\
\hline \multirow[t]{4}{*}{ Parkinson's disease (PD) } & Nurr1 & protection of dopaminergic neurons & Resting tremor \\
\hline & $\mathrm{MHC} \|$ & Immune response & \\
\hline & $\alpha$-syn & Spreading of misfolded proteins & \\
\hline & LRRK2 & Accumulation of $\alpha$-synuclein & \\
\hline Amyotrophic lateral sclerosis (ALS) & TBK1/p62 & Axonal degeneration & Immobility \\
\hline Multiple system atrophy (MSA) & PLCG/ABI3 & Immune response & Similar to early PD \\
\hline \multirow[t]{3}{*}{ Creutzfeldt-Jakob disease (CJD) } & Pro-inflammatory cytokines, etc. & Neuroinflammatory response & Compound performances \\
\hline & PGRN & Neuronal survival & \\
\hline & EAAT-1 & Neuroprotective function & \\
\hline
\end{tabular}

will further reinforce inflammasome accumulation, forming a vicious circle (Halle et al., 2008). Sequentially, $A \beta$ is degraded after the membrane extension and autophagy of microglia are initiated, which in turn will promote the abovementioned proinflammatory events (Figure 4; Cho et al., 2014; Halle et al., 2008). The microglial phagocytosis is negatively regulated by CD22 but positively regulated by TREM $2,40 \mathrm{~Hz}$ gamma entrainment using sensory stimulus (“GENUS”), and HDAC1/2 knockout (Datta et al., 2018; Martorell et al., 2019; Parhizkar et al., 2019; Pluvinage et al., 2019).

The already known effect of $A \beta$ on Wnt pathways has two aspects. One is that $A \beta$ and APP promote $\beta$-catenin phosphorylation and degradation, thus inhibiting the canonical Wnt pathway (Kim et al., 2003; Chen and Bodles, 2007; He and Shen, 2009). Tau protein is believed to stabilize $\beta$-catenin so that it can resist degradation, and the abnormal modification of tau can also cause damage to the canonical Wnt pathway (Liu et al., 2020). Another aspect is that $A \beta$ can combine with the extracellular cysteine-rich domain of Wnt receptor FZD (a receptor in the canonical pathway), and APP shares co-binding domains with Wnt co-receptor LRP6 (a receptor in the noncanonical pathways) (Magdesian et al., 2008). When LRP6 binds to the E2 domain of APP, APP will be less cleaved by secretases, thus attenuating $A \beta$ deposition (Lin et al., 2011; Liu et al., 2014; Elliott et al., 2018). Therefore, A $\beta$ has an influence not only on the canonical Wnt pathway but also on the non-canonical ones. Wnt signaling dysfunction causes $\mathrm{A} \beta$ production and synapse loss and adds difficulty to neurogenesis (He and Shen, 2009; Elliott et al., 2018). Simultaneously, $A \beta$ will induce Dkk1 production, which further inhibits the canonical Wnt pathway to exacerbate AD pathology (Killick et al., 2014). The increasing A $\beta$ burden, together with Wnt signaling dysfunction, will place great pressure to microglia. On the one hand, microglia will release various inflammatory factors when phagocytosing $A \beta$; on the other hand, Wnt signaling dysfunction will impair microglial synapse modification and promote microglial inflammation ( $\mathrm{Ru}$ et al., 2019; Song et al., 2019; Van Steenwinckel et al., 2019).

Whether the microglial function of phagocytosis will be directly injured by abnormal Wnt regulation under the double enhancement of inflammation needs to be further explored. In any case, phagocytosis is a pivotal aspect for microglia, affecting microglial neural repair, synapse modification, and inflammation profoundly. $A \beta$ and tau dissemination may be a manifestation of microglial phagocytic overload, as mentioned above. When $\mathrm{A} \beta$ phagocytosis exceeds degradation, $\mathrm{A} \beta$ is more likely to disseminate in the brain, thus enhancing inflammation. At present, ASC specks, which act as the receptor of inflammasome, are considered to be responsible for $A \beta$ dissemination (Halle et al., 2008; Freeman et al., 2017; Venegas et al., 2017). This proves that inflammation is also responsible for the dissemination of $\mathrm{AD}$ pathology, and it follows that the balance of phagocytosisdegradation is rather important for microglial functions in $\mathrm{AD}$; otherwise, microglial phagocytosis will be favorable for tau and $A \beta$ dissemination (Asai et al., 2015). The biological basis of this phenomenon is still elusive. During this process, active Wnt proteins may act as the messenger, carried by the extracellular vesicle exosome. Exosome can be secreted by cells of all kinds, including microglia (Gross et al., 2012; Hooper et al., 2012; Ibrahim et al., 2019; Kaiser et al., 2019). $\mathrm{Wn} 3 \mathrm{a}$ is able to induce exosome secretion from microglia (Hooper et al., 2012). Evidence has shown that depletion of microglia and inhibition of exosome synthesis halt tau propagation (Asai et al., 2015). Also, Wnt proteins secreted on exosomes by neurons are capable of regulating synapse numbers; however, the relationship between this regulation of synapses and microglial synapse modification is still under 


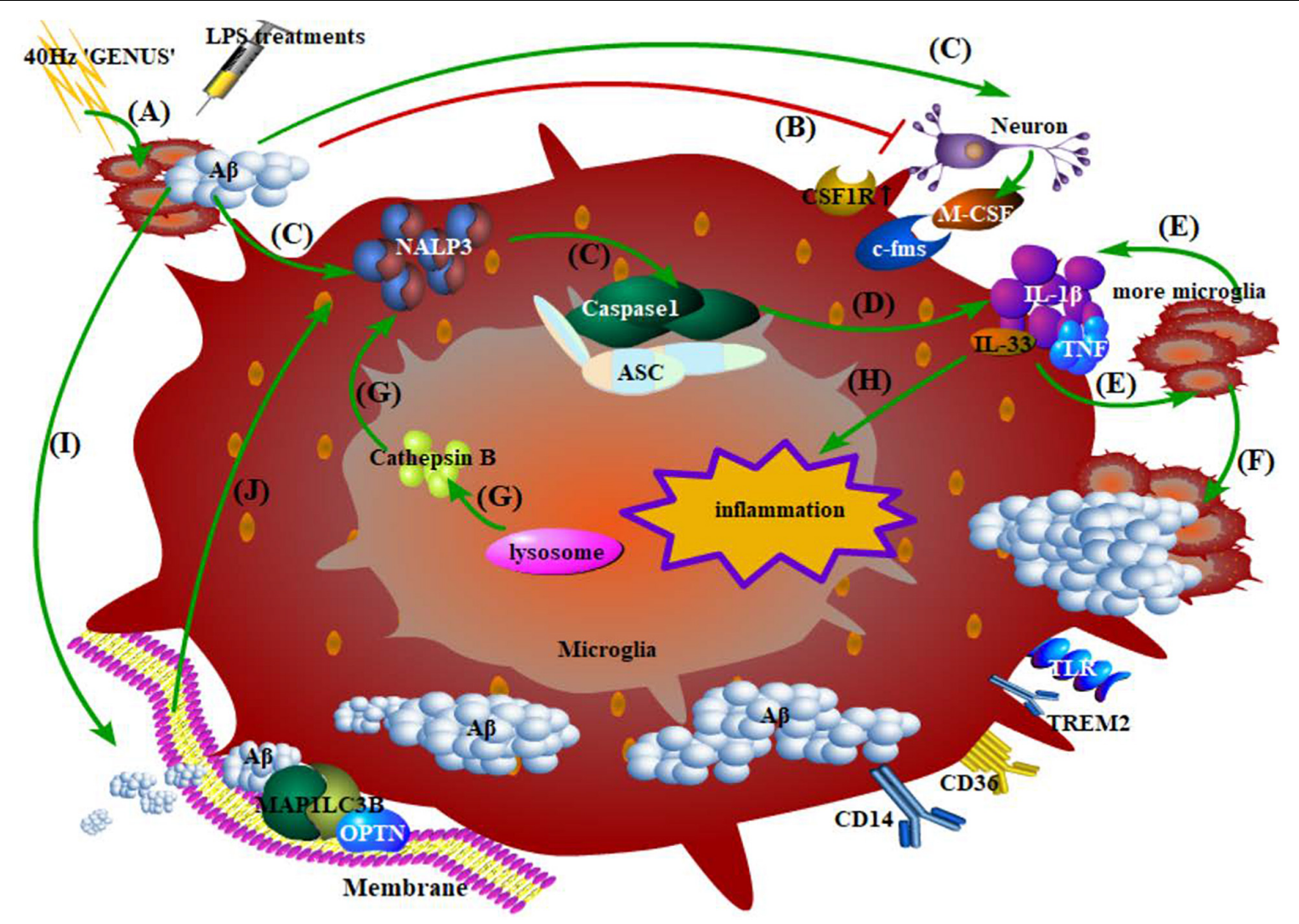

FIGURE 4 | Phagocytosis, degradation of A $\beta$ by microglia, and the inflammatory response. (A) $40 \mathrm{~Hz}$ "GENUS" induces A $\beta$ accumulation, while LPS treatments regulate immunologic memory; (B) $A \beta$ at high concentration directly damages the neurons; (C) early events of microglia-mediated inflammation: $A \beta$-induced combination of endogenous substances in neurons and their upregulated receptors on microglia, accumulation of NALP3 inflammasome, and activation of caspase-1 precursor; (D) microglia release inflammatory factors; (E) inflammatory factors recruit more microglia with more production of neurotoxic factors; (F) microglia migrate to, surround, and phagocytose AB; (G) cathepsin B released from damaged lysosomes in microglia directly reinforces NALP3 accumulation; (H) inflammatory factors produced during this period promote neuroinflammation; (I) $A \beta$ activates the initiation of autophagy and membrane extension; $A \beta$ compounds are formed and then degraded via the STK11/PRKAA1 pathway; (J) An outbreak of autophagy, in turn, promotes the accumulation of the abovementioned caspase-1 and certain inflammasomes.

investigation (Luga et al., 2012; Lee S.H. et al., 2018). This indicates the possibility that not only Wnt pathways but also Wnt proteins themselves have an influence on microglial functions. Therefore, exploring the structural connection between $A \beta$ (or tau) and Wnt proteins inside or outside microglia may further reveal the mechanism of Wnt-related microglial functions. Follow-up studies may concentrate on enhancing $\mathrm{A} \beta$ phagocytosis and degradation by microglia, balancing the two processes, preventing $\mathrm{AD}$ pathology dissemination, and antagonizing inflammation.

\section{WNT PROMOTES MICROGLIAL INFLAMMATION IN AD DEVELOPMENT}

Wnt5a (a component of non-canonical Wnt pathways) and Wnt3a (a component of the canonical Wnt pathway) have been most studied for microglial Wnt regulation. As mentioned in the Introduction section, although the canonical Wnt pathway is believed to act protectively while the non-canonical ones act deleteriously in $\mathrm{AD}$, they are both responsible for microglial inflammation. Some studies have shown that activation of the canonical Wnt pathway prevents microglial activation and alleviates neuroinflammation (Bhat et al., 2018; Song et al., 2019; Zhang D. et al., 2019). However, in primary microglia, activated Wnt-3a can upregulate $\beta$-catenin in the microglia and bring it into a pro-inflammatory state in AD (Kilander et al., 2011; Hooper et al., 2012; Halleskog and Schulte, 2013). A paradox therefore exists in that Wnt3a, as a member of the canonical Wnt pathway, which is supposed to prevent microglial inflammation in $\mathrm{AD}$, conversely promotes it. This no doubt shows the complexity of Wnt regulation in microglial inflammation. However, a deeper understanding of Wnt-related microglial inflammation targeting microglial neurogenesis, synapse modification, and phagocytosis-degradation-dissemination of $\mathrm{AD}$ pathology has not been obtained as yet. It can be speculated that the canonical Wnt pathway is capable of maintaining microglial inflammation at a level conducive to resisting AD pathology, while both canonical Wnt pathway overactivation and the 
non-canonical Wnt pathways aggravate microglial inflammation, which further exacerbates AD pathology (Dijksterhuis et al., 2015). During this process, Wnt regulation of the specific microglial functions is worth exploring, especially the noncanonical pathways, which have not been covered completely.

\section{DISCUSSION}

At present, the mechanism of $\mathrm{AD}$ has not been fully revealed, and microglial functions will need to be taken into consideration to bring the $\mathrm{AD}$ animal models used in researches much closer to the real conditions. Wnt signaling is a promising and powerful tool for resisting $\mathrm{AD}$ development. Based on the devotion of both microglia and Wnt pathways to $\mathrm{AD}$ development, future directions for $\mathrm{AD}$ pathogenesis concerning microglial inflammatory response via the Wnt pathway have been put forward in our review in sections Microglia Are More Than Immune Cells in the Central Nervous System, Microglial $\mathrm{A} \beta$ Phagocytosis-Degradation-Dissemination and Concomitant Inflammation, and Wnt promotes Microglial Inflammation in $\mathrm{AD}$ Development. Given that the microglial immuneinflammatory response, Wnt-mediated cell development, and formation of $\mathrm{AD}$ pathology are all chronic and progressive processes, figuring out the interrelations among them is of significance to further reveal the pathogenesis of $\mathrm{AD}$ and even other neurodegenerative diseases. Although functions of Wnt pathways in cancer and embryonic development have already been discovered, the influence they have on microglia and $\mathrm{AD}$ remains to be explored. What is more, the production of

\section{REFERENCES}

Aliseychik, M. P., Andreeva, T. V., and Rogaev, E. I. (2018). Immunogenetic factors of neurodegenerative diseases: the role of HLA class II. Biochemistry 83, 1104-1116. doi: 10.1134/S0006297918090122

Amal, H., Gong, G., Gjoneska, E., Lewis, S. M., Wishnok, J. S., Tsai, L. H., et al. (2019). S-nitrosylation of E3 ubiquitin-protein ligase RNF213 alters noncanonical Wnt/Ca+2 signaling in the P301S mouse model of tauopathy. Transl. Psychiatry 9:44. doi: 10.1038/s41398-019-0388-7

Arnò, B., Grassivaro, F., Rossi, C., Bergamaschi, A., Castiglioni, V., Furlan, R., et al. (2014). Neural progenitor cells orchestrate microglia migration and positioning into the developing cortex. Nat. Commun. 5:5611. doi: 10.1038/ncomms 6611

Asai, H., Ikezu, S., Tsunoda, S., Medalla, M., Luebke, J., Haydar, T., et al. (2015). Depletion of microglia and inhibition of exosome synthesis halt tau propagation. Nat. Neurosci. 18, 1584-1593. doi: 10.1038/nn.4132

Ayata, P., Badimon, A., Strasburger, H. J., Duff, M. K., Montgomery, S. E., Loh, Y. E., et al. (2018). Epigenetic regulation of brain region-specific microglia clearance activity. Nat. Neurosci. 21, 1049-1060. doi: 10.1038/s41593-0180192-3

Baas, P. W., and Black, M. M. (1990). Individual microtubules in the axon consist of domains that differ in both composition and stability. J. Cell Biol. 111, 495-509. doi: $10.1083 /$ jcb.111.2.495

Baas, P. W., and Qiang, L. (2019). Tau: it's not what you think. Trends Cell Biol. 29, 452-461. doi: 10.1016/j.tcb.2019.02.007

Baker, M., Mackenzie, I. R., Pickering-Brown, S. M., Gass, J., Rademakers, R., Lindholm, C., et al. (2006). Mutations in progranulin cause tau-negative frontotemporal dementia linked to chromosome 17. Nature 442, 916-919. doi: 10.1038 /nature05016

Barger, S. W., and Basile, A. S. (2001). Activation of microglia by secreted amyloid precursor protein evokes release of glutamate by cystine exchange and inflammatory factors from microglia is susceptible to activation of the non-canonical Wnt pathway, the mechanism of which is worth investigating. Taken together, possible subjects for further studies are as follows: (1) effects of Wnt signaling on microglial cell fate, activities, and functions; (2) effects of non-canonical Wnt pathways on microglial neuroinflammation; (3) mechanism of the shift between the canonical and non-canonical Wnt pathways and how this affects AD development; (4) whether the shift between the canonical and non-canonical Wnt pathways is responsible for the dual effects of microglial neuroinflammation in neurodegenerative diseases; (5) the balance and mechanism of phagocytosis degradation of microglia and their interplay with inflammation in $\mathrm{AD}$; (6) the biological basis and mechanism of Wnt-induced AD pathology dissemination by microglia; (7) the relation between $A \beta$ and Wnt in the protein structure; (8) Wnt-pertinent drugs and microglia-pertinent therapies for AD treatments.

\section{AUTHOR CONTRIBUTIONS}

YY and ZZ provided the idea, reviewed the documents, and completed the manuscript.

\section{FUNDING}

This work was supported by grants from the National Natural Science Foundation of China (Nos. 81822016, 81771382, and 81571249) to ZZ.

attenuates synaptic function. J. Neurochem. 76, 846-854. doi: 10.1046/j.14714159.2001.00075.x

Bellver-Landete, V., Bretheau, F., Mailhot, B., Vallières, N., Lessard, M., Janelle, M. E., et al. (2019). Microglia are an essential component of the neuroprotective scar that forms after spinal cord injury. Nat Commun. 10:518. doi: 10.1038/ s41467-019-08446-0

Bensinger, S. J., and Tontonoz, P. (2009). A Nurr1 pathway for neuroprotection. Cell 137, 26-28. doi: 10.1016/j.cell.2009.03.024

Bhat, S. A., Goel, R., Shukla, S., Shukla, R., and Hanif, K. (2018). Angiotensin receptor blockade by inhibiting glial activation promotes hippocampal neurogenesis via activation of Wnt/beta-Catenin signaling in hypertension. Mol. Neurobiol. 55, 5282-5298. doi: 10.1007/s12035-017-0754-5

Bie, B., Wu, J., Foss, J. F., and Naguib, M. (2019). Activation of mGluR1 mediates C1q-Dependent microglial phagocytosis of glutamatergic synapses in Alzheimer's rodent models. Mol. Neurobiol. 56, 5568-5585. doi: 10.1007/ s12035-019-1467-8

Böttcher, C., Schlickeiser, S., Sneeboer, M. A. M., Kunkel, D., Knop, A., Paza, E., et al. (2019). Human microglia regional heterogeneity and phenotypes determined by multiplexed single-cell mass cytometry. Nat. Neurosci. 22, 78-90. doi: 10.1038/s41593-018-0290-2

Budnik, V., Ruiz-Canada, C., and Wendler, F. (2016). Extracellular vesicles round off communication in the nervous system. Nat. Rev. Neurosci. 17, 160-172. doi: 10.1038/nrn.2015.29

Carmona-Fontaine, C., Matthews, H. K., Kuriyama, S., Moreno, M., Dunn, G. A., Parsons, M., et al. (2008). Contact inhibition of locomotion in vivo controls neural crest directional migration. Nature 456, 957-961. doi: 10.1038/ nature 07441

Chavali, M., Klingener, M., Kokkosis, A. G., Garkun, Y., Felong, S., Maffei, A., et al. (2018). Non-canonical Wnt signaling regulates neural stem cell quiescence during homeostasis and after demyelination. Nat. Commun. 9:36. doi: 10.1038/ s41467-017-02440-0 
Chen, R., Zhao, L. D., Liu, H., Li, H. H., Ren, C., Zhang, P., et al. (2017). Fluoride induces neuroinflammation and alters wnt signaling pathway in BV2 microglial cells. Inflammation 40, 1123-1130. doi: 10.1007/s10753-017-0556-y

Chen, W., Abud, E. A., Yeung, S. T., Lakatos, A., Nassi, T., Wang, J., et al. (2016). Increased tauopathy drives microglia-mediated clearance of beta-amyloid. Acta Neuropathol. Commun. 4:63. doi: 10.1186/s40478-016-0336-1

Chen, Y., and Bodles, A. M. (2007). Amyloid precursor protein modulates beta-catenin degradation. J. Neuroinflammation 4:29. doi: 10.1186/17422094-4-29

Cherubini, A., Barilani, M., Rossi, R. L., Jalal, M. M. K., Rusconi, F., Buono, G., et al. (2019). FOXP1 circular RNA sustains mesenchymal stem cell identity via microRNA inhibition. Nucleic Acids Res. 47, 5325-5340. doi: 10.1093/nar/ gkz199

Cho, M. H., Cho, K., Kang, H. J., Jeon, E. Y., Kim, H. S., Kwon, H. J., et al. (2014). Autophagy in microglia degrades extracellular beta-amyloid fibrils and regulates the NLRP3 inflammasome. Autophagy 10, 1761-1775. doi: 10.4161/ auto. 29647

Choi, S. H., Veeraraghavalu, K., Lazarov, O., Marler, S., Ransohoff, R. M., Ramirez, J. M., et al. (2008). Non-cell-autonomous effects of presenilin 1 variants on enrichment-mediated hippocampal progenitor cell proliferation and differentiation. Neuron 59, 568-580. doi: 10.1016/j.neuron.2008.07.033

Chrétien, F., Le Pavec, G., Vallat-Decouvelaere, A. V., Delisle, M. B., Uro-Coste, E., Ironside, J. W., et al. (2004). Expression of excitatory amino acid transporter1 (EAAT-1) in brain macrophages and microglia of patients with prion diseases. J. Neuropathol. Exp. Neurol. 63, 1058-1071. doi: 10.1093/jnen/63.10. 1058

Ciruna, B., Jenny, A., Lee, D., Mlodzik, M., and Schier, A. F. (2006). Planar cell polarity signalling couples cell division and morphogenesis during neurulation. Nature 439, 220-224. doi: 10.1038/nature04375

Conway, O. J., Carrasquillo, M. M., Wang, X., Bredenberg, J. M., Reddy, J. S., Strickland, S. L., et al. (2018). ABI3 and PLCG2 missense variants as risk factors for neurodegenerative diseases in Caucasians and African Americans. Mol. Neurodegener. 13:53. doi: 10.1186/s13024-018-0289-x

Crotti, A., Sait, H. R., McAvoy, K. M., Estrada, K., Ergun, A., Szak, S., et al. (2019). BIN1 favors the spreading of Tau via extracellular vesicles. Sci. Rep. 9:9477. doi: 10.1038/s41598-019-45676-0

Datta, M., Staszewski, O., Raschi, E., Frosch, M., Hagemeyer, N., Tay, T. L., et al. (2018). Histone Deacetylases 1 and 2 regulate microglia function during development, homeostasis, and neurodegeneration in a context-dependent manner. Immunity 48, 514-529.e6. doi: 10.1016/j.immuni.2018.02.016

Davies, D. S., Ma, J., Jegathees, T., and Goldsbury, C. (2017). Microglia show altered morphology and reduced arborization in human brain during aging and Alzheimer's disease. Brain Pathol. 27, 795-808. doi: 10.1111/bpa. 12456

Dejanovic, B., Huntley, M. A., De Mazière, A., Meilandt, W. J., Wu, T., Srinivasan, K., et al. (2018). Changes in the synaptic proteome in tauopathy and rescue of tau-induced synapse loss by C1q antibodies. Neuron 100, 1322-1336.e7. doi: 10.1016/j.neuron.2018.10.014

Dijksterhuis, J. P., Arthofer, E., Marinescu, V. D., Nelander, S., Uhlén, M., Pontén, F., et al. (2015). High levels of WNT5A in human glioma correlate with increased presence of tumor-associated microglia/monocytes. Exp. Cell Res. 339, 280-288. doi: 10.1016/j.yexcr.2015.10.022

Dou, Y., Wu, H. J., Li, H. Q., Qin, S., Wang, Y. E., Li, J., et al. (2012). Microglial migration mediated by ATP-induced ATP release from lysosomes. Cell Res. 22, 1022-1033. doi: 10.1038/cr.2012.10

Du Yan, S., Zhu, H., Fu, J., Yan, S. F., Roher, A., Tourtellotte, W. W., et al. (1997). Amyloid-beta peptide-receptor for advanced glycation endproduct interaction elicits neuronal expression of macrophage-colony stimulating factor: a proinflammatory pathway in Alzheimer disease. Proc. Natl. Acad. Sci. U.S.A. 94, 5296-5301. doi: 10.1073/pnas.94.10.5296

Edwards, F. A. (2019). A unifying hypothesis for Alzheimer's disease: from plaques to neurodegeneration. Trends Neurosci. 42, 310-322. doi: 10.1016/j.tins.2019. 03.003

Elliott, C., Rojo, A. I., Ribe, E., Broadstock, M., Xia, W., Morin, P., et al. (2018). A role for APP in Wnt signalling links synapse loss with beta-amyloid production. Transl. Psychiatry 8:179. doi: 10.1038/s41398-018-0231-6

Estus, S., Shaw, B. C., Devanney, N., Katsumata, Y., Press, E. E., and Fardo, D. W. (2019). Evaluation of CD33 as a genetic risk factor for Alzheimer's disease. Acta Neuropathol. 138, 187-199. doi: 10.1007/s00401-019-02000-4
Farías, G. G., Alfaro, I. E., Cerpa, W., Grabowski, C. P., Godoy, J. A., Bonansco, C., et al. (2009). Wnt5a/JNK signaling promotes the clustering of PSD-95 in hippocampal neurons. J. Biol. Chem. 284, 15857-15866. doi: 10.1074/jbc. M808986200

Filipello, F., Morini, R., Corradini, I., Zerbi, V., Canzi, A., Michalski, B., et al. (2018). The microglial innate immune receptor TREM2 is required for synapse elimination and normal brain connectivity. Immunity 48, 979-991.e8. doi: 10. 1016/j.immuni.2018.04.016

Filippini, A., Gennarelli, M., and Russo, I. (2019). alpha-synuclein and glia in Parkinson's disease: a beneficial or a detrimental duet for the endo-lysosomal system? Cell Mol. Neurobiol. 39, 161-168. doi: 10.1007/s10571-019-00649-9

Florian, M. C., Nattamai, K. J., Dörr, K., Marka, G., Uberle, B., Vas, V., et al. (2013). A canonical to non-canonical Wnt signalling switch in haematopoietic stem-cell ageing. Nature 503, 392-396. doi: 10.1038/nature12631

Freeman, L., Guo, H., David, C. N., Brickey, W. J., Jha, S., and Ting, J. P. (2017). NLR members NLRC4 and NLRP3 mediate sterile inflammasome activation in microglia and astrocytes. J. Exp. Med. 214, 1351-1370. doi: 10.1084/jem. 20150237

Fricker, M., Tolkovsky, A. M., Borutaite, V., Coleman, M., and Brown, G. C. (2018). Neuronal cell death. Physiol. Rev. 98, 813-880. doi: 10.1152/physrev.00011. 2017

Fu, A. K., Hung, K. W., Yuen, M. Y., Zhou, X., Mak, D. S., Chan, I. C., et al. (2016). IL-33 ameliorates Alzheimer's disease-like pathology and cognitive decline. Proc. Natl. Acad. Sci. U.S.A. 113, E2705-E2713. doi: 10.1073/pnas.1604032113

Füger, P., Hefendehl, J. K., Veeraraghavalu, K., Wendeln, A. C., Schlosser, C., Obermüller, U., et al. (2017). Microglia turnover with aging and in an Alzheimer's model via long-term in vivo single-cell imaging. Nat. Neurosci. 20, 1371-1376. doi: 10.1038/nn.4631

Gibson, E. M., Nagaraja, S., Ocampo, A., Tam, L. T., Wood, L. S., Pallegar, P. N., et al. (2019). Methotrexate chemotherapy induces persistent Tri-glial dysregulation that underlies chemotherapy-related cognitive impairment. Cell 176, 43-55.e13. doi: 10.1016/j.cell.2018.10.049

Giera, S., Luo, R., Ying, Y., Ackerman, S. D., Jeong, S. J., Stoveken, H. M., et al. (2018). Microglial transglutaminase-2 drives myelination and myelin repair via GPR56/ADGRG1 in oligodendrocyte precursor cells. eLife 7:e33385. doi: 10.7554/eLife.33385

Grabert, K., Michoel, T., Karavolos, M. H., Clohisey, S., Baillie, J. K., Stevens, M. P., et al. (2016). Microglial brain region-dependent diversity and selective regional sensitivities to aging. Nat. Neurosci. 19, 504-516. doi: 10.1038/nn.4222

Gray, J. D., Kholmanskikh, S., Castaldo, B. S., Hansler, A., Chung, H., Klotz, B., et al. (2013). LRP6 exerts non-canonical effects on Wnt signaling during neural tube closure. Hum. Mol. Genet. 22, 4267-4281. doi: 10.1093/hmg/ddt277

Gross, J. C., Chaudhary, V., Bartscherer, K., and Boutros, M. (2012). Active Wnt proteins are secreted on exosomes. Nat. Cell Biol. 14, 1036-1045. doi: 10.1038/ ncb2574

Gunner, G., Cheadle, L., Johnson, K. M., Ayata, P., Badimon, A., Mondo, E., et al. (2019). Sensory lesioning induces microglial synapse elimination via ADAM10 and fractalkine signaling. Nat. Neurosci. 22, 1075-1088. doi: 10.1038/s41593019-0419-y

Halle, A., Hornung, V., Petzold, G. C., Stewart, C. R., Monks, B. G., Reinheckel, T., et al. (2008). The NALP3 inflammasome is involved in the innate immune response to amyloid-beta. Nat. Immunol. 9, 857-865. doi: 10.1038/ni.1636

Halleskog, C., Mulder, J., Dahlström, J., Mackie, K., Hortobágyi, T., Tanila, H., et al. (2011). WNT signaling in activated microglia is proinflammatory. Glia 59, 119-131. doi: 10.1002/glia.21081

Halleskog, C., and Schulte, G. (2013). Pertussis toxin-sensitive heterotrimeric G(alphai/o) proteins mediate WNT/beta-catenin and WNT/ERK1/2 signaling in mouse primary microglia stimulated with purified WNT-3A. Cell. Signal. 25, 822-828. doi: 10.1016/j.cellsig.2012.12.006

Han, D., and Zhou, Y. (2019). YY1-induced upregulation of lncRNA NEAT1 contributes to $\mathrm{OGD} / \mathrm{R}$ injury-induced inflammatory response in cerebral microglial cells via Wnt/beta-catenin signaling pathway. In Vitro Cell Dev. Biol. Anim. 55, 501-511. doi: 10.1007/s11626-019-00375-y

He, P., and Shen, Y. (2009). Interruption of beta-catenin signaling reduces neurogenesis in Alzheimer's disease. J. Neurosci. 29, 6545-6557. doi: 10.1523/ JNEUROSCI.0421-09.2009

Henstridge, C. M., Hyman, B. T., and Spires-Jones, T. L. (2019). Beyond the neuron-cellular interactions early in Alzheimer disease pathogenesis. Nat. Rev. Neurosci. 20, 94-108. doi: 10.1038/s41583-018-0113-1 
Hill, R. A., Li, A. M., and Grutzendler, J. J. (2018). Lifelong cortical myelin plasticity and age-related degeneration in the live mammalian brain. Nat. Neurosci. 21, 683-695. doi: 10.1038/s41593-018-0120-6

Hooper, C., Sainz-Fuertes, R., Lynham, S., Hye, A., Killick, R., Warley, A., et al. (2012). Wnt3a induces exosome secretion from primary cultured rat microglia. BMC Neurosci. 13:144. doi: 10.1186/1471-2202-13-144

Huang, Y. A., Zhou, B., Nabet, A. M., Wernig, M., and Südhof, T. C. (2019). Differential signaling mediated by ApoE2, ApoE3, and ApoE4 in human neurons parallels Alzheimer's disease risk. J. Neurosci. 39, 7408-7427. doi: 10. 1523/JNEUROSCI.2994-18.2019

Hübner, K., Cabochette, P., Diéguez-Hurtado, R., Wiesner, C., Wakayama, Y., Grassme, K. S., et al. (2018). Wnt/beta-catenin signaling regulates VE-cadherinmediated anastomosis of brain capillaries by counteracting S1pr1 signaling. Nat. Commun. 9:4860. doi: 10.1038/s41467-018-07302-x

Ibrahim, A. G. E., Li, C., Rogers, R., Fournier, M., Li, L., Vaturi, S. D., et al. (2019). Augmenting canonical Wnt signalling in therapeutically inert cells converts them into therapeutically potent exosome factories. Nat. Biomed. Eng. 3, 695-705. doi: 10.1038/s41551-019-0448-6

Ishida-Takagishi, M., Enomoto, A., Asai, N., Ushida, K., Watanabe, T., Hashimoto, T., et al. (2012). The dishevelled-associating protein daple controls the noncanonical Wnt/Rac pathway and cell motility. Nat. Commun. 3:859. doi: 10. 1038/ncomms1861

Jiang, D. Q., Wei, M. D., Wang, K. W., Lan, Y. X., Zhu, N., and Wang, Y. (2017). Nicotine contributes to the neural stem cells fate against toxicity of microglialderived factors induced by Abeta via the Wnt/beta-catenin pathway. Int. J. Neurosci. 126, 257-268. doi: 10.3109/00207454.2015.1008696

Jiang, T., Zhang, Y. D., Gao, Q., Zhou, J. S., Zhu, X. C., Lu, H., et al. (2016). TREM1 facilitates microglial phagocytosis of amyloid beta. Acta Neuropathol. 132, 667-683. doi: 10.1007/s00401-016-1622-5

Johansson, J. U., Woodling, N. S., Wang, Q., Panchal, M., Liang, X., TruebaSaiz, A., et al. (2015). Prostaglandin signaling suppresses beneficial microglial function in Alzheimer's disease models. J. Clin. Invest. 125, 350-364. doi: 10. 1172/JCI77487

Joseph, M., Anglada-Huguet, M., Paesler, K., Mandelkow, E., and Mandelkow, E. M. (2017). Anti-aggregant tau mutant promotes neurogenesis. Mol. Neurodegener. 12:88. doi: 10.1186/s13024-017-0230-8

Kaiser, K., Gyllborg, D., Procházka, J., Salašová, A., Kompaníková, P., Molina, F. L., et al. (2019). WNT5A is transported via lipoprotein particles in the cerebrospinal fluid to regulate hindbrain morphogenesis. Nat Commun. 10:1498. doi: 10.1038/s41467-019-09298-4

Karch, C. M., and Goate, A. M. (2015). Alzheimer's disease risk genes and mechanisms of disease pathogenesis. Biol. Psychiatry 77, 43-51. doi: 10.1016/ j.biopsych.2014.05.006

Kilander, M. B., Dijksterhuis, J. P., Ganji, R. S., Bryja, V., and Schulte, G. (2011). WNT5A stimulates the GDP/GTP exchange at pertussis toxin-sensitive heterotrimeric G proteins. Cell. Signal. 23, 550-554. doi: 10.1016/j.cellsig.2010. 11.004

Killick, R., Ribe, E. M., Al-Shawi, R., Malik, B., Hooper, C., Fernandes, C., et al. (2014). Clusterin regulates beta-amyloid toxicity via Dickkopf-1 driven induction of the wnt-PCP-JNK pathway. Mol. Psychiatry 19, 88-98. doi: 10. 1038/mp.2012.163

Kim, H. S., Kim, E. M., Lee, J. P., Park, C. H., Kim, S., Seo, J. H., et al. (2003). C-terminal fragments of amyloid precursor protein exert neurotoxicity by inducing glycogen synthase kinase-3beta expression. FASEB J. 17, 1951-1953. doi: 10.1096/fj.03-0106fje

Kim, W., Khan, S. K., Gvozdenovic-Jeremic, J., Kim, Y., Dahlman, J., Kim, H., et al. (2017). Hippo signaling interactions with Wnt/beta-catenin and Notch signaling repress liver tumorigenesis. J. Clin. Invest. 127, 137-152. doi: 10.1172/ JCI88486

Kitazawa, M., Cheng, D., Tsukamoto, M. R., Koike, M. A., Wes, P. D., Vasilevko, V., et al. (2011). Blocking IL-1 signaling rescues cognition, attenuates tau pathology, and restores neuronal beta-catenin pathway function in an Alzheimer's disease model. J. Immunol. 187, 6539-6549. doi: 10.4049/jimmunol. 1100620

Klein, R. S., Garber, C., Funk, K. E., Salimi, H., Soung, A., Kanmogne, M., et al. (2019). Neuroinflammation during RNA viral infections. Annu. Rev. Immunol. 37, 73-95. doi: 10.1146/annurev-immunol-042718-041417

Klyubin, I., Walsh, D. M., Lemere, C. A., Cullen, W. K., Shankar, G. M., Betts, V., et al. (2005). Amyloid beta protein immunotherapy neutralizes Abeta oligomers that disrupt synaptic plasticity in vivo. Nat. Med. 11, 556-561. doi: 10.1038/ $\mathrm{nm} 1234$

Krasemann, S., Madore, C., Cialic, R., Baufeld, C., Calcagno, N., El Fatimy, R., et al. (2017). The TREM2-APOE pathway drives the transcriptional phenotype of dysfunctional microglia in neurodegenerative diseases. Immunity 47, 566581.e9. doi: 10.1016/j.immuni.2017.08.008

Kreutzer, A. G., and Nowick, J. S. (2018). Elucidating the structures of amyloid oligomers with macrocyclic beta-hairpin peptides: insights into Alzheimer's disease and other amyloid diseases. Acc. Chem. Res. 51, 706-718. doi: 10.1021/ acs.accounts.7b00554

Kuhn, P. H., Wang, H., Dislich, B., Colombo, A., Zeitschel, U., Ellwart, J. W., et al. (2010). ADAM10 is the physiologically relevant, constitutive alpha-secretase of the amyloid precursor protein in primary neurons. EMBO J. 29, 3020-3032. doi: 10.1038/emboj.2010.167

Lee, J. Y., Han, S. H., Park, M. H., Baek, B., Song, I. S., Choi, M. K., et al. (2018). Neuronal SphK1 acetylates COX2 and contributes to pathogenesis in a model of Alzheimer's disease. Nat. Commun. 9:1479. doi: 10.1038/s41467-018-03674-2

Lee, S. H., Shin, S. M., Zhong, P., Kim, H. T., Kim, D. I., Kim, J. M., et al. (2018). Reciprocal control of excitatory synapse numbers by Wnt and Wnt inhibitor PRR7 secreted on exosomes. Nat. Commun. 9:3434. doi: 10.1038/s41467-01805858-2

Li, Y., Du, X. F., Liu, C. S., Wen, Z. L., and Du, J. L. (2012). Reciprocal regulation between resting microglial dynamics and neuronal activity in vivo. Dev. Cell 23, 1189-1202. doi: 10.1016/j.devcel.2012.10.027

Lin, C., Lu, W., Zhai, L., Bethea, T., Berry, K., Qu, Z., et al. (2011). Mesd is a general inhibitor of different Wnt ligands in Wnt/LRP signaling and inhibits PC-3 tumor growth in vivo. FEBS Lett. 585, 3120-3125. doi: 10.1016/j.febslet. 2011.08.046

Liu, C. C., Tsai, C. W., Deak, F., Rogers, J., Penuliar, M., Sung, Y. M., et al. (2014). Deficiency in LRP6-mediated Wnt signaling contributes to synaptic abnormalities and amyloid pathology in Alzheimer's disease. Neuron 84, 63-77. doi: 10.1016/j.neuron.2014.08.048

Liu, E., Zhou, Q., Xie, A. J., Li, X., Li, M., Ye, J., et al. (2020). Tau acetylates and stabilizes beta-catenin thereby promoting cell survival. EMBO Rep. 21:e48328. doi: $10.15252 /$ embr.201948328

Liu, Y., Walter, S., Stagi, M., Cherny, D., Letiembre, M., Schulz-Schaeffer, W., et al. (2005). LPS receptor (CD14): a receptor for phagocytosis of Alzheimer's amyloid peptide. Brain 128(Pt 8), 1778-1789. doi: 10.1093/brain/awh531

Llorens, F., López-González, I., Thüne, K., Carmona, M., Zafar, S., Andréoletti, O., et al. (2014). Subtype and regional-specific neuroinflammation in sporadic creutzfeldt-jakob disease. Front. Aging Neurosci. 6:198. doi: 10.3389/fnagi.2014. 00198

Long, H., Zhong, G., Wang, C., Zhang, J., Zhang, Y., Luo, J., et al. (2019). TREM2 Attenuates Abeta1-42-Mediated neuroinflammation in BV-2 cells by downregulating TLR signaling. Neurochem. Res. 44, 1830-1839. doi: 10.1007/ s11064-019-02817-1

Lucin, K. M., O’Brien, C. E., Bieri, G., Czirr, E., Mosher, K. I., Abbey, R. J., et al. (2013). Microglial beclin 1 regulates retromer trafficking and phagocytosis and is impaired in Alzheimer's disease. Neuron 79, 873-886. doi: 10.1016/j.neuron. 2013.06.046

Luga, V., Zhang, L., Viloria-Petit, A. M., Ogunjimi, A. A., Inanlou, M. R., Chiu, E., et al. (2012). Exosomes mediate stromal mobilization of autocrine Wnt-PCP signaling in breast cancer cell migration. Cell 151, 1542-1556. doi: 10.1016/j. cell.2012.11.024

Lui, H., Zhang, J., Makinson, S. R., Cahill, M. K., Kelley, K. W., Huang, H. Y., et al. (2016). Progranulin deficiency promotes circuit-specific synaptic pruning by microglia via complement activation. Cell 165, 921-935. doi: 10.1016/j.cell. 2016.04.001

Ma, S., Meng, Z., Chen, R., and Guan, K. L. (2019). The hippo pathway: biology and pathophysiology. Annu. Rev. Biochem. 88, 577-604. doi: 10.1146/annurevbiochem-013118-111829

Magdesian, M. H., Carvalho, M. M., Mendes, F. A., Saraiva, L. M., Juliano, M. A., Juliano, L., et al. (2008). Amyloid-beta binds to the extracellular cysteine-rich domain of Frizzled and inhibits Wnt/beta-catenin signaling. J. Biol. Chem. 283, 9359-9368. doi: 10.1074/jbc.M707108200

Markovinovic, A., Cimbro, R., Ljutic, T., Kriz, J., Rogelj, B., and Munitic, I. (2017). Optineurin in amyotrophic lateral sclerosis: Multifunctional adaptor protein at the crossroads of different neuroprotective mechanisms. Prog. Neurobiol. 154, 1-20. doi: 10.1016/j.pneurobio.2017.04.005 
Martin, E., Boucher, C., Fontaine, B., and Delarasse, C. (2017). Distinct inflammatory phenotypes of microglia and monocyte-derived macrophages in Alzheimer's disease models: effects of aging and amyloid pathology. Aging Cell 16, 27-38. doi: 10.1111/acel.12522

Martin, L., Latypova, X., Wilson, C. M., Magnaudeix, A., Perrin, M. L., and Terro, F. (2013a). Tau protein phosphatases in Alzheimer's disease: the leading role of PP2A. Ageing Res. Rev. 12, 39-49. doi: 10.1016/j.arr.2012. 06.008

Martin, L., Latypova, X., Wilson, C. M., Magnaudeix, A., Perrin, M. L., Yardin, C., et al. (2013b). Tau protein kinases: involvement in Alzheimer's disease. Ageing Res. Rev. 12, 289-309. doi: 10.1016/j.arr.2012.06.003

Martorell, A. J., Paulson, A. L., Suk, H. J., Abdurrob, F., Drummond, G. T., Guan, W., et al. (2019). Multi-sensory gamma stimulation ameliorates Alzheimer'sAssociated pathology and improves cognition. Cell 177, 256-271.e22. doi: 10. 1016/j.cell.2019.02.014

Matias, D., Dubois, L. G., Pontes, B., Rosário, L., Ferrer, V. P., Balça-Silva, J., et al. (2019). GBM-Derived Wnt3a induces M2-Like phenotype in microglial cells through Wnt/beta-catenin signaling. Mol. Neurobiol. 56, 1517-1530. doi: 10.1007/s12035-018-1150-5

Meilandt, W. J., Ngu, H., Gogineni, A., Lalehzadeh, G., Lee, S. H., Srinivasan, K., et al. (2020). Trem 2 deletion reduces late-stage amyloid plaque accumulation, elevates the Abeta42:Abeta40 ratio, and exacerbates axonal dystrophy and dendritic spine loss in the PS2APP Alzheimer's mouse model. J. Neurosci. 40, 1956-1974. doi: 10.1523/JNEUROSCI.1871-19.2019

Morizane, A., Kikuchi, T., Hayashi, T., Mizuma, H., Takara, S., Doi, H., et al. (2017). MHC matching improves engraftment of iPSC-derived neurons in non-human primates. Nat. Commun. 8:385. doi: 10.1038/s41467-017-00926-5

Mrdjen, D., Pavlovic, A., Hartmann, F. J., Schreiner, B., Utz, S. G., Leung, B. P., et al. (2018). High-Dimensional single-cell mapping of central nervous system immune cells reveals distinct myeloid subsets in health, aging, and disease. Immunity 48, 380-395. doi: 10.1016/j.immuni.2018.02.014

Nagarajan, N., Jones, B. W., West, P. J., Marc, R. E., and Capecchi, M. R. (2018). Corticostriatal circuit defects in Hoxb8 mutant mice. Mol. Psychiatry 23, 18681877. doi: 10.1038/mp.2017.180

Neniskyte, U., Neher, J. J., and Brown, G. C. (2011). Neuronal death induced by nanomolar amyloid beta is mediated by primary phagocytosis of neurons by microglia. J. Biol. Chem. 286, 39904-39913. doi: 10.1074/jbc.M111.267583

O’Koren, E. G., Yu, C., Klingeborn, M., Wong, A. Y. W., Prigge, C. L., Mathew, R., et al. (2019). Microglial function is distinct in different anatomical locations during retinal homeostasis and degeneration. Immunity 50, 723-737.e7. doi: 10.1016/j.immuni.2019.02.007

Olmos-Alonso, A., Schetters, S. T., Sri, S., Askew, K., Mancuso, R., VargasCaballero, M., et al. (2016). Pharmacological targeting of CSF1R inhibits microglial proliferation and prevents the progression of Alzheimer's-like pathology. Brain 139(Pt 3), 891-907. doi: 10.1093/brain/awv379

Ouali Alami, N., Schurr, C., Olde Heuvel, F., Tang, L., Li, Q., Tasdogan, A., et al. (2018). NF-kappaB activation in astrocytes drives a stage-specific beneficial neuroimmunological response in ALS. EMBO J. 37:e98697. doi: 10.15252/embj. 201798697

Paolicelli, R. C., Jawaid, A., Henstridge, C. M., Valeri, A., Merlini, M., Robinson, J. L., et al. (2017). TDP-43 depletion in microglia promotes amyloid clearance but also induces synapse loss. Neuron 95, 297-308.e6. doi: 10.1016/j.neuron. 2017.05.037

Parhizkar, S., Arzberger, T., Brendel, M., Kleinberger, G., Deussing, M., Focke, C., et al. (2019). Loss of TREM2 function increases amyloid seeding but reduces plaque-associated ApoE. Nat. Neurosci. 22, 191-204. doi: 10.1038/s41593-0180296-9

Patel, A. K., Park, K. K., and Hackam, A. S. (2017). Wnt signaling promotes axonal regeneration following optic nerve injury in the mouse. Neuroscience 343, 372-383. doi: 10.1016/j.neuroscience.2016.12.020

Pester, O., Barrett, P. J., Hornburg, D., Hornburg, P., Pröbstle, R., Widmaier, S., et al. (2013). The backbone dynamics of the amyloid precursor protein transmembrane helix provides a rationale for the sequential cleavage mechanism of gamma-secretase. J. Am. Chem. Soc. 135, 1317-1329. doi: 10. 1021/ja3112093

Petrache, A. L., Rajulawalla, A., Shi, A., Wetzel, A., Saito, T., Saido, T. C., et al. (2019). Aberrant excitatory-inhibitory synaptic mechanisms in entorhinal cortex microcircuits during the pathogenesis of Alzheimer's disease. Cereb. Cortex 29, 1834-1850. doi: 10.1093/cercor/bhz016
Pluvinage, J. V., Haney, M. S., Smith, B. A. H., Sun, J., Iram, T., Bonanno, L., et al. (2019). CD22 blockade restores homeostatic microglial phagocytosis in ageing brains. Nature 568, 187-192. doi: 10.1038/s41586-019-1088-4

Ren, D. N., Chen, J., Li, Z., Yan, H., Yin, Y., Wo, D., et al. (2015). LRP5/6 directly bind to Frizzled and prevent Frizzled-regulated tumour metastasis. Nat. Commun. 6:6906. doi: 10.1038/ncomms7906

Robakis, N. K., Wisniewski, H. M., Jenkins, E. C., Devine-Gage, E. A., Houck, G. E., Yao, X. L., et al. (1987). Chromosome 21q21 sublocalisation of gene encoding beta-amyloid peptide in cerebral vessels and neuritic (senile) plaques of people with Alzheimer disease and Down syndrome. Lancet 1, 384-385. doi: 10.1016/s0140-6736(87)91754-5

Robinson, J. L., Molina-Porcel, L., Corrada, M. M., Raible, K., Lee, E. B., Lee, V. M., et al. (2014). Perforant path synaptic loss correlates with cognitive impairment and Alzheimer's disease in the oldest-old. Brain 137(Pt 9), 2578-2587. doi: 10.1093/brain/awu190

Roostaei, T., Nazeri, A., Felsky, D., De Jager, P. L., Schneider, J. A., Pollock, B. G., et al. (2017). Genome-. (wide)interaction study of brain beta-amyloid burden and cognitive impairment in Alzheimer's disease. Mol. Psychiatry 22, 287-295. doi: 10.1038/mp.2016.35

Ross, S. P., Baker, K. E., Fisher, A., Hoff, L., Pak, E. S., and Murashov, A. K. (2018). miRNA-431 prevents amyloid-beta-induced synapse loss in neuronal cell culture model of Alzheimer's disease by silencing kremenl. Front. Cell Neurosci. 12:87. doi: 10.3389/fncel.2018.00087

Rosso, S. B., Sussman, D., Wynshaw-Boris, A., and Salinas, P. C. (2005). Wnt signaling through Dishevelled, Rac and JNK regulates dendritic development. Nat. Neurosci. 8, 34-42. doi: 10.1038/nn1374

Ru, W., Liu, X., Bae, C., Shi, Y., Walikonis, R., Mo Chung, J., et al. (2019). Microglia mediate HIV-1 gp120-Induced synaptic degeneration in spinal pain neural circuits. J. Neurosci. 39, 8408-8421. doi: 10.1523/JNEUROSCI.2851-18.2019

Rui, Q., Ni, H., Li, D., Gao, R., and Chen, G. (2018). The role of LRRK2 in neurodegeneration of parkinson disease. Curr. Neuropharmacol. 16, 13481357. doi: 10.2174/1570159X16666180222165418

Sakae, N., Heckman, M. G., Vargas, E. R., Carrasquillo, M. M., Murray, M. E., Kasanuki, K., et al. (2019). Evaluation of associations of Alzheimer's disease risk variants that are highly expressed in microglia with neuropathological outcome measures. J. Alzheimers Dis. 70, 659-666. doi: 10.3233/JAD-190451

Sayed, F. A., Telpoukhovskaia, M., Kodama, L., Li, Y., Zhou, Y., Le, D., et al. (2018). Differential effects of partial and complete loss of TREM2 on microglial injury response and tauopathy. Proc. Natl. Acad. Sci. U.S.A. 115, 10172-10177. doi: $10.1073 /$ pnas. 1811411115

Schafer, D. P., Heller, C. T., Gunner, G., Heller, M., Gordon, C., Hammond, T., et al. (2016). Microglia contribute to circuit defects in Mecp2 null mice independent of microglia-specific loss of Mecp2 expression. eLife 5:e15224. doi: 10.7554/eLife.15224

Schafer, D. P., Lehrman, E. K., Kautzman, A. G., Koyama, R., Mardinly, A. R., Yamasaki, R., et al. (2012). Microglia sculpt postnatal neural circuits in an activity and complement-dependent manner. Neuron 74, 691-705. doi: 10. 1016/j.neuron.2012.03.026

Scholz, B., Korn, C., Wojtarowicz, J., Mogler, C., Augustin, I., Boutros, M., et al. (2016). Endothelial RSPO3 controls vascular stability and pruning through non-canonical WNT/Ca(2+)/NFAT signaling. Dev. Cell 36, 79-93. doi: 10.1016/ j.devcel.2015.12.015

Sellers, K. J., Elliott, C., Jackson, J., Ghosh, A., Ribe, E., Rojo, A. I., et al. (2018). Amyloid beta synaptotoxicity is Wnt-PCP dependent and blocked by fasudil. Alzheimers Dement. 14, 306-317. doi: 10.1016/j.jalz.2017.09.008

Sellgren, C. M., Gracias, J., Watmuff, B., Biag, J. D., Thanos, J. M., Whittredge, P. B., et al. (2019). Increased synapse elimination by microglia in schizophrenia patient-derived models of synaptic pruning. Nat. Neurosci. 22, 374-385. doi: 10.1038/s41593-018-0334-7

Shankar, G. M., Li, S., Mehta, T. H., Garcia-Munoz, A., Shepardson, N. E., Smith, I., et al. (2008). Amyloid-beta protein dimers isolated directly from Alzheimer's brains impair synaptic plasticity and memory. Nat. Med. 14, 837-842. doi: $10.1038 / \mathrm{nm} 1782$

Shen, Q., Cohen, B., Zheng, W., Rahbar, R., Martin, B., Murakami, K., et al. (2017). Notch shapes the innate immunophenotype in breast cancer. Cancer Discov. 7, 1320-1335. doi: 10.1158/2159-8290.CD-17-0037

Shi, Y., Yamada, K., Liddelow, S. A., Smith, S. T., Zhao, L., Luo, W., et al. (2017). ApoE4 markedly exacerbates tau-mediated neurodegeneration in a mouse model of tauopathy. Nature 549, 523-527. doi: 10.1038/nature24016 
Sierra, A., Encinas, J. M., Deudero, J. J., Chancey, J. H., Enikolopov, G., OverstreetWadiche, L. S., et al. (2010). Microglia shape adult hippocampal neurogenesis through apoptosis-coupled phagocytosis. Cell Stem Cell. 7, 483-495. doi: 10. 1016/j.stem.2010.08.014

Slotkin, J. R., Pritchard, C. D., Luque, B., Ye, J., Layer, R. T., Lawrence, M. S., et al. (2017). Biodegradable scaffolds promote tissue remodeling and functional improvement in non-human primates with acute spinal cord injury. Biomaterials 123, 63-76. doi: 10.1016/j.biomaterials.2017.01.024

Song, D., Zhang, X., Chen, J., Liu, X., Xue, J., Zhang, L., et al. (2019). Wnt canonical pathway activator TWS119 drives microglial anti-inflammatory activation and facilitates neurological recovery following experimental stroke. J. Neuroinflammation 16:256. doi: 10.1186/s12974-019-1660-8

Stancu, I. C., Cremers, N., Vanrusselt, H., Couturier, J., Vanoosthuyse, A., Kessels, S., et al. (2019). Aggregated Tau activates NLRP3-ASC inflammasome exacerbating exogenously seeded and non-exogenously seeded Tau pathology in vivo. Acta Neuropathol. 137, 599-617. doi: 10.1007/s00401-018-01957-y

Toledo, E. M., and Inestrosa, N. C. (2010). Activation of Wnt signaling by lithium and rosiglitazone reduced spatial memory impairment and neurodegeneration in brains of an APPswe/PSEN1DeltaE9 mouse model of Alzheimer's disease. Mol. Psychiatry 15, 272-285. doi: 10.1038/mp.2009.72

Trillaud-Doppia, E., and Boehm, J. (2018). The amyloid precursor protein intracellular domain is an effector molecule of metaplasticity. Biol. Psychiatry 83, 406-415. doi: 10.1016/j.biopsych.2016.12.015

Turano, E., Busetto, G., Marconi, S., Guzzo, F., Farinazzo, A., Commisso, M., et al. (2015). Neurotoxicity and synaptic plasticity impairment of $\mathrm{N}$-acetylglucosamine polymers: implications for Alzheimer's disease. Neurobiol. Aging 36, 1780-1791. doi: 10.1016/j.neurobiolaging.2014.12.033

Ueno, M., Fujita, Y., Tanaka, T., Nakamura, Y., Kikuta, J., Ishii, M., et al. (2013). Cortical neurons require microglial support for survival during postnatal development. Nat. Neurosci. 16, 543-551. doi: 10.1038/nn.3358

Ulland, T. K., Song, W. M., Huang, S. C., Ulrich, J. D., Sergushichev, A., Beatty, W. L., et al. (2017). TREM2 maintains microglial metabolic fitness in Alzheimer's disease. Cell 170, 649-663.e13. doi: 10.1016/j.cell.2017.07.023

Van Steenwinckel, J., Schang, A. L., Krishnan, M. L., Degos, V., Delahaye-Duriez, A., Bokobza, C., et al. (2019). Decreased microglial Wnt/beta-catenin signalling drives microglial pro-inflammatory activation in the developing brain. Brain 142, 3806-3833. doi: 10.1093/brain/awz319

Venegas, C., Kumar, S., Franklin, B. S., Dierkes, T., Brinkschulte, R., Tejera, D., et al. (2017). Microglia-derived ASC specks cross-seed amyloid-beta in Alzheimer's disease. Nature 552, 355-361. doi: 10.1038/nature25158

Vogel, A., Upadhya, R., and Shetty, A. K. (2018). Neural stem cell derived extracellular vesicles: attributes and prospects for treating neurodegenerative disorders. EBioMedicine 38, 273-282. doi: 10.1016/j.ebiom.2018.11.026

Wang, J. Z., Grundke-Iqbal, I., and Iqbal, K. (1996). Glycosylation of microtubuleassociated protein tau: an abnormal posttranslational modification in Alzheimer's disease. Nat. Med. 2, 871-875. doi: 10.1038/nm0896-871

Watson, A. L., Rahrmann, E. P., Moriarity, B. S., Choi, K., Conboy, C. B., Greeley, A. D., et al. (2013). Canonical Wnt/beta-catenin signaling drives human schwann cell transformation, progression, and tumor maintenance. Cancer Discov. 3, 674-689. doi: 10.1158/2159-8290.CD-13-0081

Wendeln, A. C., Degenhardt, K., Kaurani, L., Gertig, M., Ulas, T., Jain, G., et al. (2018). Innate immune memory in the brain shapes neurological disease hallmarks. Nature 556, 332-338. doi: 10.1038/s41586-018-0023-4

Wheeler, D. L., Sariol, A., Meyerholz, D. K., and Perlman, S. (2018). Microglia are required for protection against lethal coronavirus encephalitis in mice. J. Clin. Invest. 128, 931-943. doi: 10.1172/JCI97229

Wiendl, H., Feger, U., Mittelbronn, M., Jack, C., Schreiner, B., Stadelmann, C., et al. (2005). Expression of the immune-tolerogenic major histocompatibility molecule HLA-G in multiple sclerosis: implications for CNS immunity. Brain 128(Pt 11), 2689-2704. doi: 10.1093/brain/awh609

Willem, M., Tahirovic, S., Busche, M. A., Ovsepian, S. V., Chafai, M., Kootar, S., et al. (2015). $\eta$-Secretase processing of APP inhibits neuronal activity in the hippocampus. Nature 526, 443-447. doi: 10.1038/nature14864
Wlodarczyk, A., Holtman, I. R., Krueger, M., Yogev, N., Bruttger, J., Khorooshi, R., et al. (2017). A novel microglial subset plays a key role in myelinogenesis in developing brain. EMBO J. 36, 3292-3308. doi: 10.15252/embj.201696056

Xia, S., Ji, R., and Zhan, W. (2017). Long noncoding RNA papillary thyroid carcinoma susceptibility candidate 3 (PTCSC3) inhibits proliferation and invasion of glioma cells by suppressing the Wnt/beta-catenin signaling pathway. BMC Neurol. 17:30. doi: 10.1186/s12883-017-0813-6

Xiong, X. Y., Liu, L., and Yang, Q. W. (2016). Functions and mechanisms of microglia/macrophages in neuroinflammation and neurogenesis after stroke. Prog. Neurobiol. 142, 23-44. doi: 10.1016/j.pneurobio.2016.05.001

Yang, G., Meng, Y., Li, W., Yong, Y., Fan, Z., Ding, H., et al. (2011). Neuronal MCP-1 mediates microglia recruitment and neurodegeneration induced by the mild impairment of oxidative metabolism. Brain Pathol. 21, 279-297. doi: 10.1111/j.1750-3639.2010.00445.x

Yao, Y. Y., Bian, L. G., Yang, P., Sui, Y., Li, R., Chen, Y. L., et al. (2019). Gastrodin attenuates proliferation and inflammatory responses in activated microglia through Wnt/beta-catenin signaling pathway. Brain Res. 1717, 190-203. doi: 10.1016/j.brainres.2019.04.025

Yin, C., Ackermann, S., Ma, Z., Mohanta, S. K., Zhang, C., Li, Y., et al. (2019). ApoE attenuates unresolvable inflammation by complex formation with activated C1q. Nat. Med. 25, 496-506. doi: 10.1038/s41591-018-0336-8

Yoshiyama, Y., Higuchi, M., Zhang, B., Huang, S. M., Iwata, N., Saido, T. C., et al. (2007). Synapse loss and microglial activation precede tangles in a P301S tauopathy mouse model. Neuron 53, 337-351. doi: 10.1016/j.neuron.2007. 01.010

Zhang, D., Lu, Z., Man, J., Cui, K., Fu, X., Yu, L., et al. (2019). Wnt-3a alleviates neuroinflammation after ischemic stroke by modulating the responses of microglia/macrophages and astrocytes. Int. Immunopharmacol. 75:105760. doi: 10.1016/j.intimp.2019.105760

Zhang, L., Fang, Y., Cheng, X., Lian, Y. J., and Xu, H. L. (2019). Silencing of long noncoding RNA SOX21-AS1 relieves neuronal oxidative stress injury in mice with Alzheimer's disease by upregulating FZD3/5 via the Wnt signaling pathway. Mol. Neurobiol. 56, 3522-3537. doi: 10.1007/s12035-018-1299-y

Zhang, Z., Hartmann, H., Do, V. M., Abramowski, D., Sturchler-Pierrat, C., Staufenbiel, M., et al. (1998). Destabilization of beta-catenin by mutations in presenilin-1 potentiates neuronal apoptosis. Nature 395, 698-702. doi: 10.1038/ 27208

Zhang, Z., Li, X. G., Wang, Z. H., Song, M., Yu, S. P., Kang, S. S., et al. (2018). deltaSecretase-cleaved Tau stimulates Abeta production via upregulating STAT1BACE1 signaling in Alzheimer's disease. Mol. Psychiatry [Epub ahead of print]. doi: 10.1038/s41380-018-0286-Z

Zhang, Z., Song, M., Liu, X., Kang, S. S., Kwon, I. S., Duong, D. M., et al. (2014). Cleavage of tau by asparagine endopeptidase mediates the neurofibrillary pathology in Alzheimer's disease. Nat. Med. 20, 1254-1262. doi: 10.1038/nm. 3700

Zheng, H., Jia, L., Liu, C. C., Rong, Z., Zhong, L., Yang, L., et al. (2017). TREM2 promotes microglial survival by activating Wnt/beta-catenin pathway. J. Neurosci. 37, 1772-1784. doi: 10.1523/JNEUROSCI.2459-16.2017

Zhou, J., Yu, W., Zhang, M., Tian, X., Li, Y., and Lü, Y. (2019). Imbalance of microglial TLR4/TREM2 in LPS-Treated APP/PS1 transgenic mice: a potential link between Alzheimer's disease and systemic inflammation. Neurochem. Res. 44, 1138-1151. doi: 10.1007/s11064-019-02748-x

Conflict of Interest: The authors declare that the research was conducted in the absence of any commercial or financial relationships that could be construed as a potential conflict of interest.

Copyright (c) 2020 Yang and Zhang. This is an open-access article distributed under the terms of the Creative Commons Attribution License (CC BY). The use, distribution or reproduction in other forums is permitted, provided the original author(s) and the copyright owner(s) are credited and that the original publication in this journal is cited, in accordance with accepted academic practice. No use, distribution or reproduction is permitted which does not comply with these terms. 